\title{
Experimental demonstration of a new concept of drag reduction and thermal protection for hypersonic vehicles
}

\author{
Zonglin Jiang · Yunfeng Liu • Guilai Han · Wei Zhao
}

Received: 16 January 2009 / Accepted: 12 March 2009 / Published online: 16 April 2009

(C) The Chinese Society of Theoretical and Applied Mechanics and Springer-Verlag GmbH 2009

\begin{abstract}
A new idea of drag reduction and thermal protection for hypersonic vehicles is proposed based on the combination of a physical spike and lateral jets for shockreconstruction. The spike recasts the bow shock in front of a blunt body into a conical shock, and the lateral jets work to protect the spike tip from overheating and to push the conical shock away from the blunt body when a pitching angle exists during flight. Experiments are conducted in a hypersonic wind tunnel at a nominal Mach number of 6. It is demonstrated that the shock/shock interaction on the blunt body is avoided due to injection and the peak pressure at the reattachment point is reduced by $70 \%$ under a $4^{\circ}$ attack angle.
\end{abstract}

Keywords Hypersonic vehicle - Shock-reconstruction · Drag reduction - Thermal protection - Spike and lateral jets

\section{Introduction}

For hypersonic vehicles, the shock drag comprises two thirds of the total cruise drag [1], therefore, some methods were proposed to reduce it. One of them is to install a physical spike on the nose of blunt bodies and was considered as an effective means for shock-drag reduction [2-5]. The spike recasts a bow shock into a conical shock and induces a low pressure region ahead of blunt bodies. Therefore, approximate 50\%

The project supported by the National Natural Science Foundation of China (10632090) and the National Aerodynamic Pre-Research Foundation (513130401).

Z. Jiang $(\varangle) \cdot$ Y. Liu $\cdot$ G. Han $\cdot$ W. Zhao

Key Laboratory of High Temperature Gas Dynamics, Institute of Mechanics, Chinese Academy of Sciences, 100190 Beijing, China

e-mail: zljiang@imech.ac.cn drag reduction was predicted under the condition of a zero attack angle. However, the spiked-nose blunt body structure becomes ineffective for shock-drag reduction if the attack angle is not zero [4] because that the separation region in the windward side becomes much smaller, and the conical shock and the reattachment shock interact with each other on the shoulder of blunt bodies, which results in the extremely high pressure at the interaction point, and it is much higher than the stagnation pressure. Moreover, the huge aerodynamic heating at both the spike tip and the interaction point on blunt bodies blocks the way for spike application to hypersonic vehicles.

In order to achieve efficient wave drag reduction under non-zero attack angles and avoid the severe aerodynamic heating problem, a new concept was proposed based on the idea that the conical shock angle should be enlarged to push the conical shock away from the blunt body surface and make the shock/shock interaction point occur in space not at the blunt body. Doing so, a spike and lateral jets are combined together to develop a shock-reconstructing system for hypersonic vehicles. When the spike recasts the bow shock in front of a blunt body into a conical shock, the lateral jets work to protect the spike tip from overheating and push the conical shock away from the blunt body when a pitching angle exists during flight. Experiments were conducted in a hypersonic wind tunnel for conceptual demonstration, and the relevant results from flow visualization and pressure measurement are discussed briefly in this note.

\section{Experimental setup}

The test model consists of a hemisphere and a cylinder being $D=80 \mathrm{~mm}$ in diameter, is actually a spiked-nose hemisphere-cylinder. The spike diameter $d$ is $12 \mathrm{~mm}$ and 


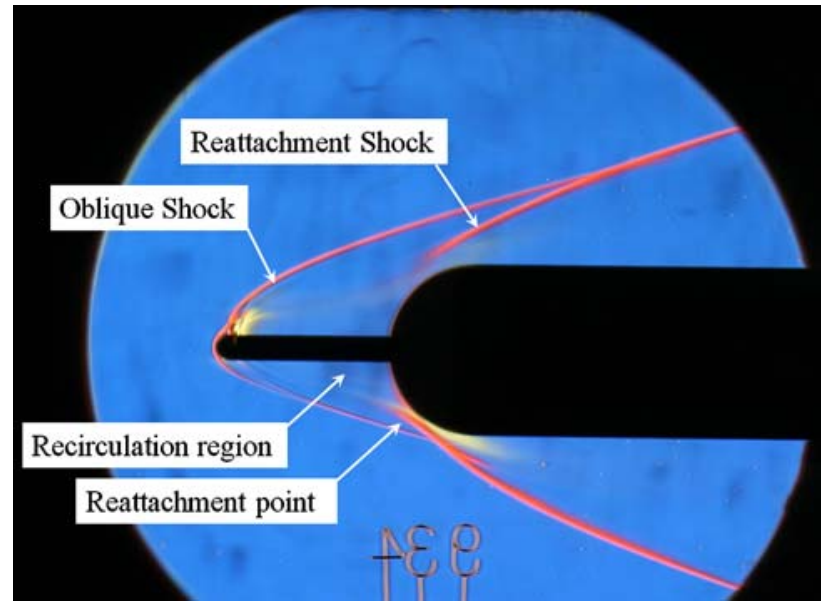

Fig. 1 Schlieren photograph of the experiment at a zero attack angle with air injection only in the upper half of the test model

$d / D=0.15$. The spike length $L$ is $80 \mathrm{~mm}$ and $L / D=1.0$. A circular orifice being $1.0 \mathrm{~mm}$ in width is located nearby the spike tip for a lateral injection, as shown in Fig. 1. Fifteen pressure transducers are distributed along the model generatrix starting from the spike tip. Flow visualization and pressure measurement were conducted by using a $\Phi 500 \mathrm{~mm}$ hypersonic wind tunnel in China Academy of Aerospace and Aerodynamics. The test flow is at a nominal Mach number of six, the total pressure of $2.0 \mathrm{MPa}$ and a Reynolds number of $1.98 \times 10^{7}$. The total pressure for lateral air jets is $0.6 \mathrm{MPa}$.

\section{Results and discussion}

Figure 1 shows a schlieren photograph of flow visualization when the test model is at a zero attack angle, and the bow shock in the blunt body is recast into a conical shock by the spike and lateral jets. By examining Fig. 1, it is observable that the conical shock in the upper half of the photograph was pushed away from the blunt body by air injection, and the conical shock in the low half interacts with the reattached shock at the blunt body shoulder.

Figure 2 presents two pressure distributions along the model generatrix, one with a lateral injection and one without it. The abscissa is the ratio of the distance along the body generatrix from the stagnation point to the body diameter. The peak pressure with lateral injection is about $9,000 \mathrm{~Pa}$, while the peak pressure at the interaction location without lateral injection arises up to $26,000 \mathrm{~Pa}$. As a result, the peak pressure is reduced by $66 \%$ and the lateral injection effect was well demonstrated.

Schlieren photographs of the experiments with $\pm 4^{\circ}$ attack angles are presented in Fig. 3. From Fig. 3a one can find that the conical shock and the reattached bow shock inter-

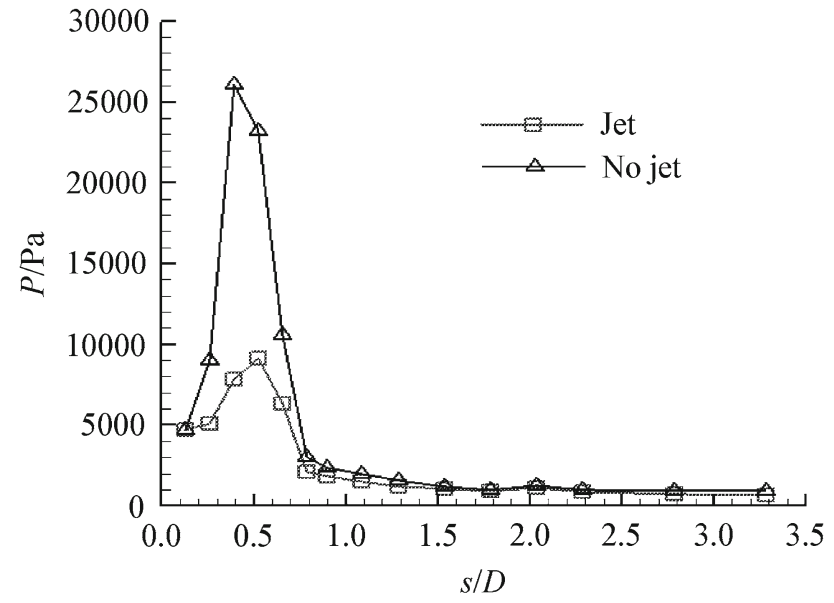

Fig. 2 Pressure distributions along the generatrix of the blunt body at a zero attack angle

act on the blunt body shoulder, which results in an extremely higher pressure at the interaction point and also aerodynamic heating. Figure $3 \mathrm{~b}$ shows that the recast shock structure by the lateral jet from the experiment with a $4^{\circ}$ attack angle. It is observable that the conical shock angel is enlarged and the interaction point is pushed away from the blunt body due to lateral injection.

The comparison of the pressure distributions along the blunt body generatrix with and without lateral jets under $\pm 4^{\circ}$ attack angle are plotted together in Fig. 4. It can be seen that the pressure peak without lateral injection is about $85,000 \mathrm{~Pa}$, and even higher than the stagnation pressure of 59,300 $\mathrm{Pa}$ for the blunt body without any spikes under the same test condition. This shows that the spike becomes ineffective under the condition of non-zero attack angles, and also indicates severe aerodynamic heating. However, if applying lateral injection, the peak pressure is greatly reduced to about $25,000 \mathrm{~Pa}$, and a $70 \%$ reduction was achieved. Moreover, the aerodynamic heating could also be expected to reduce greatly. This result demonstrated that the new concept is still effective for shock drag reduction thermal protection even hypersonic vehicles in a pitching flight condition.

\section{Conclusions}

In this note, experimental results are reported to demonstrate the validity of a new idea for drag reduction and thermal protection of hypersonic vehicles. The spike recasts the bow shock in front of a blunt body into a conical shock, which could reduce shock drag and lowers the thermal aerodynamic environment around hypersonic vehicles. Lateral jets work to push the conical shock away from the blunt body when a pitching angle exists during flight, and achieve 
Fig. 3 Schlieren photographs for two experiments at the same condition but with different attack angles. a Experiment with $\mathrm{a}-4^{\circ}$ attack angle. b Experiment with a $4^{\circ}$ attack angle
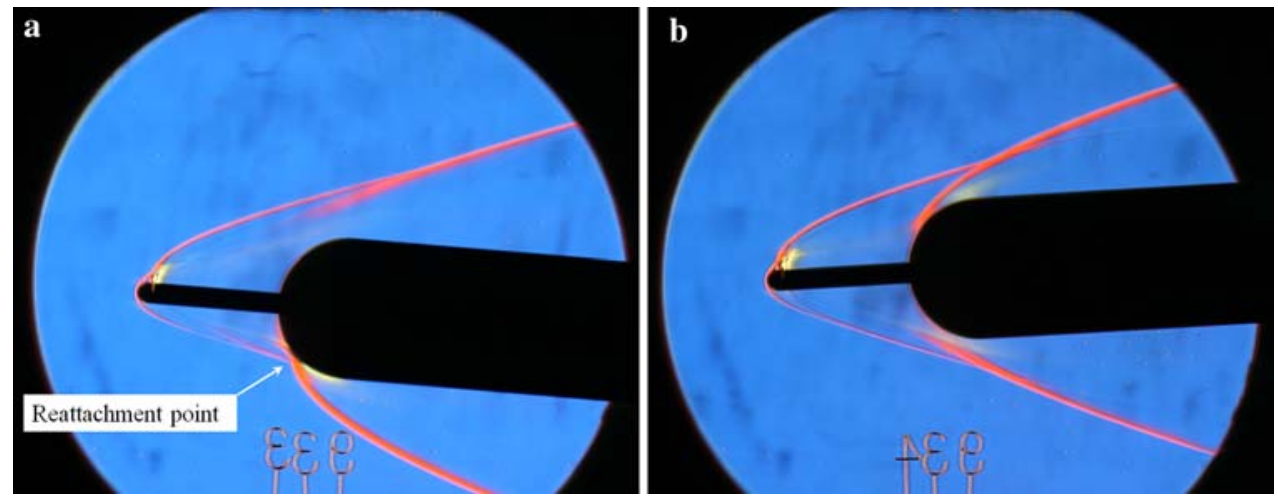

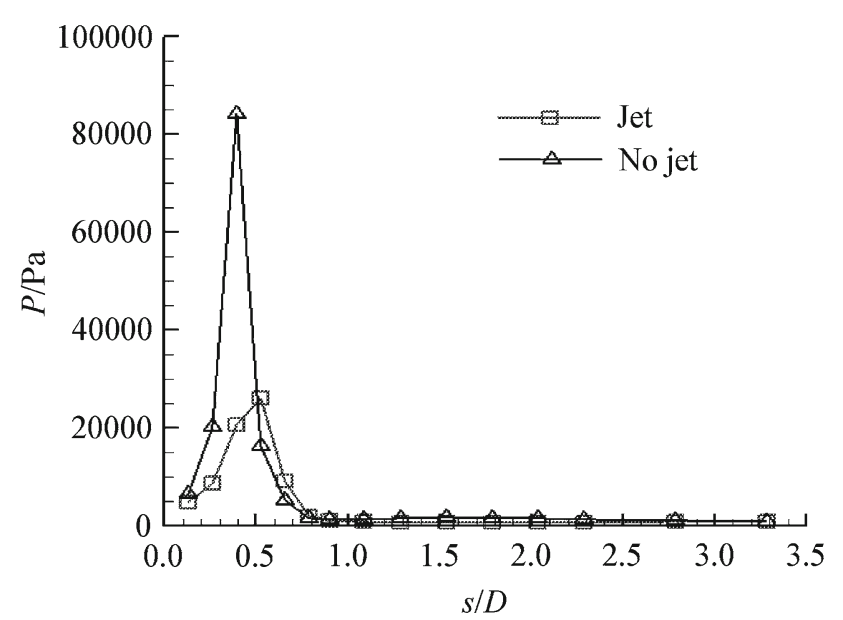

Fig. 4 Pressure distributions along the generatrix of the test model at $\pm 4^{\circ}$ attack angles

efficient cooling of the spike tip so that a long hypersonic flight can be realized. The flow visualization and the pressure measurement results presented in this note strongly support the conclusions, and the new concept was well demonstrated to be of potential importance for hypersonic vehicles.

\section{References}

1. Bushnell, D.M.: Shock wave drag reduction. Annu. Rev. Fluid. Mech. 36, 81-96 (2004)

2. Crawford, D.H.: Investigation of the flow over a spiked-nose hemisphere-cylinder at a Mach number of 6.8. NASA TN-D118 (1959)

3. Hutt, C.R., Howe, A.J.: Forward facing spike effects of bodies of different cross section in supersonic flow. Aeronaut. J. R. Aeronaut. Soc. 93(6), 229-234 (1989)

4. Milićev, S.S., Pavlović, M.D., Ristić, S., Vitić, A.: On the influence of spike shape at supersonic flow past blunt bodies. Facta Univ. Ser. Mech. Autom. Control Robot. 3(12), 371-382 (2002)

5. Menezes, V., Saravanan, S., Jagadeesh, G., Reddy, K.P.J.: Experimental investigations of hypersonic flow over highly blunted cones with aerospikes. AIAA J. 41(10), 1955-1961 (2003) 